\title{
SOME CLOSING THOUGHTS
}

\author{
H. M. Van Horn \\ Department of Physics and Astronomy \\ University of Rochester \\ Rochester, NY 14627-0011, USA
}

\section{Introduction}

I am very pleased to be the final speaker at this conference, for it gives me the pleasant opportunity to do several special things.

First, I would like to take this opportunity to thank Academician B. Kovachev, Prof. K. Panov, the other members of the Local Organizing Committee, and the Astronomy Section of the Bulgarian Academy of Sciences for the superb local arrangements. You have been excellent hosts, extending us a gracious welcome, treating us royally, and providing for our every need.

I would also like to thank Professors Georges Michaud and Alexander Tutukov and the other members of the Scientific Organizing Committee for conceiving the idea for this conference. Your insight that the time was ripe for such a meeting, crossing the artifical boundaries we usually erect in the H-R diagram, has been proven correct. This conference has helped us to overcome the myopia we each feel from concentrating on our own small corners of astronomy, expanding our scientific horizons back to the entire H-R diagram.

As I have listened to each of you speaking about your own areas of specialization and making "the photospheric abundance connections" in many different ways, I have also been impressed with the international nature of this effort. We have heard talks from astronomers whose native languages are Bulgarian, German, Russian, Spanish, French, and many others. This prompts me to say to you now: "Az ze izviniavim che ne moga da govoria bulgarski!"1

In trying to think how best to summarize the results of this exceptionally diverse conference, I decided in the end to adapt a format used by a very distinguished astronomer in another meeting on a similarly broad topic. With apologies to Allan Sandage (1987), my remarks are accordingly designed to leave you with some useful (?) definitions, some memorable (?) quotations, and some remaining homework problems.

\footnotetext{
1 "I apologize that I do not speak Bulgarian!"
} 


\section{Some Useful (?) Definitions}

During the course of this meeting, we learned that the abbreviation "SN" has several possible meanings, depending on who was speaking. The ones I remember are (1) Signalto-Noise (several people), (2) SuperNova (Nomoto), (3) Superficially Normal (Cowley), and Solar Neighborhood (Truran).

We also learned that the abbreviation "S," especially when used to denote a particular reference, can mean either Sackman or Scalo or Smith. We can now add Sakhibullin and Sion, among others. We also learned that the proper way to use this abbreviation is to cite all the references beginning with this first initial using this same abbreviation!

\section{Some Memorable (?) Quotations}

I have also been taking careful notes of remarks made by one or another of you that seemed to me to be particularly memorable. I'd like to remind you of some of those quotations.

It seems to me especially appropriate to begin with W. P. Bidelman's comment, which was quoted to us by Charles Cowley, as it captures the spirit of this conference remarkably well. Bidelman said, "I do not know, nor have I ever seen, a normal A0 star!"

John Landstreet reminded us why the study of stars with unusual compositions is important. He said, "Peculiar stars are not the 'freak show' of stellar evolution; they are the keys to understanding normal $A$ and $B$ stars."

V. Ivanov put it more picturesquely when he referred to "... the stellar 'strip-tease' known as the Wolf-Rayet phenomenon ..." As a self-proclaimed newcomer to the field of stellar evolution, Ivanov gave us all a chuckle when he introduced himself by asking rhetorically "Who is this Ivanov?"

Another quotation that aroused a considerable reaction was N. A. Sakhibullin's assertion "LTE is for lazy people!". Not everyone agreed with this. A. J. Willis explained why when he told us that "One WN model requires about 20 hours on a Cray XMP!" The reward for doing such sophisticated non-LTE models is very great, however: "The difference between the models and the observations is too small to see." (R. P. Kudritzki, quoted by A. J. Willis)

We also learned of some new aspects of time measurement at this meeting. David Lambert in his remarks in the previous summary talk spoke of "... years AI (After Iben)." Presumably this refers to time measured after one of Icko's seminal early papers!

Another aspect of time appeared in Jim Truran's talk, when he remarked "When the Galaxy was one day old, it already knew the rules we're still trying to infer!"

Perhaps my favorite quotation from this meeting, however, was one that occurred on the very first day. When we had some temporary difficulty darkening the lecture hall so that viewgraph projections could be seen, N. Grevesse I think captured the attraction for all of us of the bright sunshine and crashing surf just outside when he exclaimed "If we had known we couldn't get the shade down, we could have stayed at the beach!" 


\section{Some Remaining "Homework" Problems}

At the very beginning of our conference, I began taking note of some of the open questions that were raised in your various talks. I confess to recording those which seemed to me either particularly interesting or important, and I apologize to those of you whose favorite puzzle I have omitted. When I began to prepare this talk, I also realized that I had listed far too many topics to cover in the time available. Fortunately, Professors Baschek and Lambert have done admirable jobs in the preceding summary lectures, and they have already covered many of the topics I had listed. I have therefore relegated to an Appendix a mere listing of the topics I shall not discuss. For my own contribution to these summary talks, I have tried to begin more-or-less in the AGB phase and to continue on from there to the endpoints of stellar evolution. I have also gone on at the end of the resulting list of "homework problems" to some of the larger questions on which our meeting also touched. In each case, I have listed the name of the speaker in whose talk the question was raised, and I strongly recommend referring to the corresponding papers earlier in this volume for more information. Any misrepresentations of the issues are entirely my own fault, however.

It seems to me that one of the nicest overviews of the subject of our meeting was provided by the figure that John Landstreet showed in his lecture. This diagram, taken from the review by Vauclair and Vauclair (1982), shows the locations in the H-R diagram of the many different chemically peculiar stars we have been discussing. I shall not repeat the figure here, but I recommend it, particularly for non-specialists like myself, as an excellent way to become oriented in this complex and often confusing maze of abundance anomalies.

The following is the list of problems I have compiled from your presentations:

\subsection{IS THE ${ }^{12} \mathrm{C}(\alpha, \gamma){ }^{16} \mathrm{O}$ CROSS-SECTION FINALLY KNOWN? (QUESTION FROM FLOOR; SACKMAN, ARNOULD)}

The rate of the ${ }^{12} \mathrm{C}(\alpha, \gamma){ }^{16} \mathrm{O}$ reaction still appears to be uncertain by as much as a factor of two. This rate plays a critical role in our understanding of the $\mathrm{C} / \mathrm{O}$ ratio in stars. It affects the evolution of low-mass stars and nucleosynthesis on the AGB, the subsequent cooling of white dwarfs, and the development of presupernova models of massive stars. Thus, it is important to continue efforts to reduce the uncertainties in this critical reaction rate.

\subsection{HOW CAN SUPER-Li-RICH AGB STARS BE MADE? (VAUCLAIR)}

Some AGB stars are observed to have $\mathrm{Li}$ abundanaces up to ten times the cosmic abundance value for this element. It must therefore be possible to produce $\mathrm{Li}$ in these stars. One way to do this may be for a deep surface convection zone to extend down to temperatures $T \sim 3 \times 10^{7} \mathrm{~K}$, where the ${ }^{3} \mathrm{He}(\alpha, \gamma){ }^{7} \mathrm{Be}$ reaction occurs. The convection zone would bring the ${ }^{7} \mathrm{Be}$ to the surface, where it would decay into ${ }^{7} \mathrm{Li}$. However, the same convection zone should also return the ${ }^{7} \mathrm{Li}$ to regions of such high temperatures that it would be destroyed again. For this reason, as Sylvie Vauclair pointed out, the process of Li-production in AGB stars needs to be reconsidered. 
To understand s-process nucleosynthesis in low-mass AGB stars, it is necessary to know the neutron source. David Lambert described to us how spectroscopists are currently trying to constrain values for the neutron number density by determining the isotopic ratio of ${ }^{95} \mathrm{Zr}$ to ${ }^{96} \mathrm{Zr}$ from the $\mathrm{ZrO}$ bands. In order to use this technique successfully, however, it is essential to have a substantial amount of information about the spectrum of this molecule, either from laboratory measurements or from numerical computations. This is just one example of the urgent need for much more information about molecular spectra.

\subsection{HOW DO ZAEHB STARS FINISH THEIR EVOLUTION? (cf. HEBER)}

Ulli Heber defined the "zero-age extended horizontal branch" (ZAEHB) as consisting of objects that have just begun core He-burning and which have $\mathrm{H}$ envelopes that are too thin to sustain H-burning shells. The hot sdB stars appear to form a homogeneous group that lie in the region of the $\left(\log g, \log T_{\text {eff }}\right)$ diagram through which the ZAEHB models are expected to evolve. The evolutionary connection to subsequent phases, however, is not yet clear, except that we expect such objects ultimately to evolve into white dwarfs.

\subsection{WHAT ARE THE sdO STARS? (cf. HEBER, TUTUKOV)}

In contrast to the $\mathrm{sdB}$ stars, the sdO stars do not form a homogeneous group. Some seem to have evolved from the EHB, while others appear to have originated on the AGB. Alternatively, the sdOs may have resulted from mergers of binaries in which both members of the pair were originally degenerate He white dwarfs (cf. Iben and Tutukov 1989). It would be very nice to know exactly how these stars were formed!

\subsection{IS THE [Fe/H] DEFICIENCY IN POST-AGB SUPERGIANTS EXPLAINED BY DIFFUSION? (BOND)}

Low-mass post-AGB stars are difficult to distinguish from higher-mass stars which are in earlier phases of evolution. One possible discriminant is the $[\mathrm{Fe} / \mathrm{H}] \mathrm{ratio}$, which can place constraints on the progenitor mass: low values of $[\mathrm{Fe} / \mathrm{H}]$ suggest great age and thus low progenitor mass. Howard Bond told us about one such star that has $[\mathrm{Fe} / \mathrm{H}]$ as low as -3.2 or -4.5 . Is this exceptionally low value caused by diffusion of the heavy elements into the interior of the star? Alternatively, is it due to differential loss in a wind?

Or is there some other explanation?

\subsection{WE NEED MORE DETAILED NLTE ANALYSES OF sdOs AND CENTRAL STARS OF PLANETARY NEBULAE (CSPN), WITH WINDS! ( $c$. HEBER, MENDEZ)}

Analyses of the spectra of the very hot sdO stars and CSPN demand non-LTE calculations. The CSPN may also have substantial mass-loss rates. Computations of synthetic spectra for hot stars with strong winds are exceedingly difficult, and the computing power necessary to perform such calculations has only become available to a few groups within the past several years. Some comparisons between simpler hydrostatic, NLTE, plane-parallel models seem 
to show reasonable agreement, but the uncertainties in interpretation can only be removed by constructing more detailed models.

\subsection{WHY DO SOME PG1159-035 STARS PULSATE, WHILE OTHERS WITH IDEN- TICAL $T_{\text {eff }}, \log g$, AND SURFACE COMPOSITION DO NOT? ( $c$. HEBER)}

This question is one of the major puzzles concerning the PG1159 (GW Vir) stars. For example, four stars in this spectroscopic class appear to have exactly the same surface composition and $\log g$. Of these four, the stars PG1159-035 and PG1520+525 both have effective temperatures $T_{\text {eff }}=140,000 \mathrm{~K}$, but only the first exhibits pulsations. Similarly, PG1707 +427 and PG1424 +535 both have $T_{\text {eff }}=100,000 \mathrm{~K}$, but again only the first of these pulsates. An understanding of the reasons for the differences between the pulsating and non-pulsating stars will give us considerable information about these stars.

\subsection{IS THE ENVELOPE MASS OF HELIUM IN THE PG1159-035 STARS AS LARGE AS THE EVOLUTIONARY CALCULATIONS SUGGEST? (LIEBERT)}

Stellar evolution calculations yield values $M(\mathrm{He}) \sim 10^{-2} M_{\odot}$ for the mass of the He-layer in the hottest white dwarfs (the PG1159-035 stars). This is approximately the minimum mass of He that can undergo nuclear burning. However, if the He-layer mass is actually this large, it is difficult to understand the subsequent spectral evolution of the white dwarfs, as Jim Liebert explained. For example, there is increasing evidence that mixing between the surface $\mathrm{H}$ layer and the deeper He layer produces "He- pollution," but not He domination, in white dwarfs cooler than $\sim 11,000 \mathrm{~K}$. This is hard to understand if $M(\mathrm{He})$ is as large as the evolutionary calculations suggest. A similar problem occurs in the interpretation of "C-pollution" in the spectra of the DQ white dwarfs, where $M(\mathrm{He}) \sim 10^{-3.5} M_{\odot}$ has been inferred to account for the observed abundance of the dredged-up carbon. It is conceivable that much of the $\mathrm{H}$ or He layer mass can be lost by winds in the hottest pre-white-dwarf stars, but quantitative investigations of this possibility have not yet been performed.

\subsection{WHAT IS THE MASS OF HYDROGEN IN WHITE DWARFS? (cf. D'ANTONA, LIEBERT, FONTAINE, KOESTER)}

A similar problem occurs with hydrogen. Evolutionary calculations yield $M(\mathrm{H}) \sim 10^{-4} M_{\odot}$. However, some white dwarf pulsation calculations require $M(\mathrm{H}) \lesssim 10^{-8} M_{\odot}$ in order to reproduce the observed "blue edge" of the instability strip, and a similar value is necessary if mixing between the $\mathrm{H}$ and He layers is to produce the "red edge." Even more extreme values, $M(\mathrm{H}) \sim 10^{-15} M_{\odot}$, are found if $\mathrm{H} / \mathrm{He}$ mixing is responsible for producing the DB stars at temperatures $T_{\text {eff }} \lesssim 20,000 \mathrm{~K}$. The most extreme values suggested so far are $M(\mathrm{H}) \sim 10^{-18} M_{\odot}$, which may be necessary to produce compositionally stratified atmospheres for the DAO or DAB white dwarfs. The resolution of this question has the possibility of placing very significant constraints on the structure and evolution of the earliest pre-white-dwarf phases. 
We now understand quite a bit about the evolution of stars of large and intermediate mass, but the evolution of low-mass stars is in some ways more difficult to calculate. In particular, it is extremely difficult to model the process of ejection of a planetary. The generally accepted scenario is that a low-mass star climbs the AGB, with the rate of climb increasing as the star increases in luminosity, and eventually leaves the AGB, probably while still burning hydrogen. The increasing UV flux ionizes the nebula. We are still not sure whether the separation between the remnant star and the nebula occurs on the low$T_{\text {eff }}$ side of the H-R diagram, however, or whether it happens as the star moves toward the white dwarf cooling track. Is PN ejection the result of a "superwind" as the star ascends the AGB, is it caused by a thermal pulse, or is there some other mechanism?

\subsection{TWO PROBLEMS WITH THE DAO STARS IN PLANETARY NEBULAE (KOESTER)}

In his discussion of compositional stratification in white dwarf atmospheres, Detlev Koester highlighted two significant problems:

4.12.1: $T_{\mathrm{eff}} \sim 60,000 \mathrm{~K}$ is not hot enough to ionize the nebula.

and

4.12.2: The white dwarf cooling time is much longer than the nebular expansion time.

At least half of the CSPN appear to be DAO white dwarfs, all of which appear to have $T_{\text {eff }} \sim 50-70,000 \mathrm{~K}$. These contain detectable amounts of He, as the spectral type implies. However, even $\mathrm{He}$ abundances as low as $\mathrm{He} / \mathrm{H} \sim 10^{-3}$ are enough to "kill" essentially all the EUV flux shortward of $\lambda 228 \AA$. Thus, such stars should not be able to ionize the surrounding nebulae, in contradiction to the observations. Clearly, both of these problems could be solved if the true effective temperature were much higher than $\sim 60,000 \mathrm{~K}$. However, then we have the difficulty of explaining how a very hot star can masquerade as one that appears much cooler. Perhaps here again models of very hot, NLTE atmospheres with winds will help to resolve the problems.

\subsection{WHAT IS THE CORE COMPOSTION OF G117-B15A FROM II? (D'ANTONA)}

Franca D'Antona told us about recent observations of the pulsations of the ZZ Ceti white dwarf G117-B15A, which have determined the timescale of period change to be $\Pi / \dot{\Pi}=$ $(8.2 \pm 5.0) \times 10^{8}$ years. This is much faster than expected from the most detailed evolutionary models for cooling $\mathrm{C} / \mathrm{O}$ white dwarfs and suggests a core composition of larger atomic mass, which would cool more rapidly. However, ONeMg compositions are thought to be produced only during C-burning, which requires white dwarf masses of 1.2 to $1.37 M_{\odot}$. This is more than twice as large as the mean mass of the white dwarfs. Is our understanding of white dwarf cooling flawed? Alternatively, is there something we do not understand about the 
conditions for the production of heavy nuclei in the pre-white dwarf phases? Or is the observed period change in this white dwarf due to some other cause?

\subsection{WE NEED MORE NLTE ANALYSES FOR WR STARS, WITH WINDS! (cf. WILLIS)}

In his discussion of abundances in the Wolf-Rayet stars, A. J. Willis reminded us that the atmospheres of these stars are dominated by winds, with mass-loss rates reaching $10^{-5}$ to $10^{-4} M_{\odot} \mathrm{yr}^{-1}$ and with temperatures $T_{\text {eff }} \sim 30-70,000 \mathrm{~K}$. He emphasized that it is not possible to use traditional model atmospheres to analyze the spectra of these stars. The most detailed NLTE line-transfer models are currently being computed by the groups at JILA and in Kiel; both are producing line profiles that closely resemble the observations. Because of the large amount of computing time required for each model ( $c f$. the quotation from Willis in section 3), the number of detailed comparisons between models and observations has so far been quite limited. To increase our understanding of the abundances in these stars - and to interpret the evolutionary clues they contain - we clearly need more such calculations.

\subsection{WE NEED DETAILED CLUSTER/MODEL-ISOCHRONE COMPARISONS FOR MASSIVE STARS (MAEDER)}

Andre Maeder pointed out that the evolution of massive stars is quite sensitive to the heavy element abundance $Z$. For example, the rate of mass loss varies as $\dot{M} \propto Z^{\alpha}$, and low $Z$ also gives very different $C / O$ ratios than are produced with $Z \sim 0.04$. Such changes affect the chemical yields needed for galactic evolution studies, and the yields may differ with position in a galaxy as well as with time. Thus it is very important to test the theoretical models by comparing detailed isochrone calculations for massive stars with cluster observations. The discovery that the progenitor of supernova $1987 \mathrm{~A}$ was a blue supergiant, in contrast to pre-supernova expectations, illustrates the importance and value of such tests.

\subsection{WHAT IS HAPPENING TO $[\mathrm{O} / \mathrm{Fe}]$ FOR $[\mathrm{Fe} / \mathrm{H}] \lesssim-2$ ? (TRURAN)}

In his discussion of the chemical evolution of galaxies, Jim Truran emphasized that it is very important to determine how $[\mathrm{O} / \mathrm{Fe}]$ behaves for the most metal-deficient stars (those with $[\mathrm{Fe} / \mathrm{H}] \lessgtr-2)$. The $[0 / \mathrm{Fe}]$ ratio is determined by the $r$-process in stars with $M>10 M_{\odot}$, and the $r$-process is the dominant one in most metal-deficient stars. There may or may not be a trend to large values of $[0 / \mathrm{Fe}]$ as $[\mathrm{Fe} / \mathrm{H}]$ decreases $(c f$. Wheeler, Sneden, and Truran 1989 and references therein). If such a trend exists, it may have important implications for the ages of the globular clusters. It could also be a signature of very massive early stars. Our understanding of the early evolution of galaxies will be substantially improved by the resolution of such issues.

\subsection{IS THE QUARK-HADRON TRANSITION A FIRST-ORDER TRANSITION? (REEVES)}

In the very first talk at this conference, Hubert Reeves described some new results concerning the "Big Bang," which set the stage for the subsequent formation of galaxies and stars. 
He pointed out that the assumption of spatial homgeneity, employed in the "standard Big Bang," may be wrong if the transition that forms hadrons in the quark-gluon plasma is a first-order phase transition. He and his colleagues have already begun to work out some of the consequences of the spatial inhomogeneities that result from the assumption that this is a first-order phase transition. They find that with such inhomogeneities it is possible to fit the abundance data for ${ }^{3} \mathrm{He}, \mathrm{Li}$, etc., with $0.01<\Omega_{b}<0.2$, and that with some "fine tuning" it may even be possible to go to $\Omega_{b}=1$. Because of the profound cosmological consequences this would have, it is clearly very important to determine whether or not the process of hadron formation does occur via a first-order phase transition.

\section{Acknowledgements}

The author's participation in this conference was made possible by support from the U.S. National Science Foundation under grant AST 88-20322, which he gratefully acknowledges.

\section{Appendix: Some Additional Homework Problems}

Most of these were discussed rather thoroughly in the preceding two summary talks, so I just list them here for completeness.

A.1: Why are elements with $\chi>10 \mathrm{eV}$ depleted by factors of about 4.5 in the solar corona? (cf. Grevesse)

A.2: What causes the $\lambda$ Boötis stars? (Baschek and others)

A.3: Determinations of the Fe abundacnes are badly needed in AmFm stars! (Michaud)

A.4: Why is the "Li gap" found in the Hyades and Ursa Major but not in younger clusters? (cf. Rebolo)

A.5: Why does the ${ }^{12} \mathrm{C} /{ }^{13} \mathrm{C}$ ratio vary with $M / M_{\odot}$ for $M<2.5 M_{\odot}$ ? (cf. Sneden)

A.6: How are abundance anomalies produced in Ap stars and some horizontal branch stars? What does the magnetic field do? (Baschek, after Ryabchikova)

A.7: Do HgMn stars develop by first pushing up $\mathrm{P}$ and then $\mathrm{Mn}$ ? (Cowley)

A.8: Is there a real $\mathrm{Na}$ problem? (Ivanov)

A.9: What causes gradual enrichment of He with age in main sequence B stars? (Lyubimkov)

A.10: We need more maps of abundance anomalies and magnetic fields from Doppler shifts and polarizations! (cf. Landstreet)

A.11: Can $\sigma$ Ori B teach us about pulsar magnetospheres? (Van Horn, after Hunger)

A.12: Why do $\mathrm{CN}$ variations occur down the giant branch all the way to the main sequence? ( $c f$. Sneden)

\section{References}

Iben, I., and Tutukov, A. V. (1989) 'Model Stars with Degenerate Dwarf Cores and Helium-Burning Shells: A Stationary-Burning Approximation', Ap. J., 342, 430. 
Sandage, A. (1987) 'Summary - Population II Stars' in IAU Coll. No. 95: The Second Conference on Faint Blue Stars, A. G. D. Philip, D. S. Hayes, and J. W. Liebert (eds.), L. Davis Press, Schenectady, p. 469.

Vauclair, S., and Vauclair, G. (1982) 'Element Segretation in Stellar Outer Layers', Ann. Rev. Astron. Astrophys., 20, 37.

Wheeler, J. C., Sneden, C., and Truran, J. W. (1989) 'Abundance Ratios as a Function of Metallicity' Ann. Rev. Astron. Astrophys., 27, 279. 